\title{
On Generalizations of Isoparity
}

\author{
L. C. Btedenharn*, J. Nuyts** and H. RuegG \\ CERN, Geneva
}

Received December 22, 1965

\begin{abstract}
The generalization of the isoparity ( $G$-parity) for an arbitrary internal symmetry group is the problem of adjoining appropriately the charge conjugation operation, thereby extending the group. A complete solution to this problem is given, and explicitly detailed for the four families of compact, simple and simplyconnected Lie groups $\left(S U_{n}, \bar{R}_{2 k+1}, \bar{R}_{2 k}\right.$ and $\left.S p_{n}\right)$.

The extended isoparity is shown to depend upon the structure of the groups in question, and the required structure is developed and summarized. The properties of the extended isoparity are discussed and two special cases - 'strong $G$-parity' and 'weak $G$-parity' - are treated in more detail.
\end{abstract}

\section{Introduction and summary}

The charge conjugation operation, $\mathscr{C}$, - more properly "matterantimatter conjugation" - is a symmetry operation of quantum mechanics which briefly put, reverses the sign of all (generalized) charges for all states:

$$
\mathscr{C}\left|A\left(Q_{i}\right)\right\rangle=\eta_{A}\left|A\left(-Q_{i}\right)\right\rangle
$$

where $\eta_{A}$ indicates the intrinsic charge conjugation parity of the state $|A\rangle$ and $\left|\eta_{A}\right|^{2}=1$.

We suppose moreover that there exists a continuous compact Lie group which acts on the states $|A\rangle$. For the special case where only ordinary charge and the isospin group are considered the operation of charge reversal, i.e., reflection in a plane containing the charge axis, can be combined in the well-known way [1] with a rotation $(C)$ in isospace to yield a reflection operator reversing all directions in isospace; this is the usual one of the two possible isoparity operators (the $G$ parity [2] (see also references in [2]) to use the customary but less descriptive term) defined as $G=\eta_{A} C \mathscr{C}$.

The present paper is concerned with the problem of generalizing the concept of the isoparity operator from the special example of the isospin group of internal symmetries to the larger class of internal symmetries

* National Science Foundation Senior Postdoctoral Fellow on leave from Duke University, Durham, North Carolina, U. S. A.

** N. A.T. O. Postdoctoral Fellow. A part of this work was done at the Institute for Advanced Study (Princeton) supported by a grant of the Air Force Office of Scientific Research, Office of Aerospace Research, United States Air Force, under AFOSR Nr 42.65 
given by the unitary, rotation and symplectic families of groups (more accurately their covering groups). Although only a very few cases of these groups are seriously considered in the literature at present (notably $S U_{3}, S U_{4}$ and $S U_{6}$ ) it is none the less desirable to see the general features of the isoparity problem. This is particularly valuable since the isoparity problem may be seen itself as a special, and instructive, example of the more general problem of group extensions discussed by MICHEL [3] in his Istanbul lecture (1962); in more physical language this is the problem of determining inter-relations between the generalized charges, spins and discrete operations. The isoparity problem is very much simpler than this general problem in that only the compact internal symmetry groups are considered (complications of the Poincaré group do not enter for example) and only a single operator $(G)$ is adjoined (rather than the complete set of all possible discrete invariances). Owing to these simplifications a complete and explicit answer can be given.

Let us first note that a particular basis in the compact continuous group can always be chosen such that, on the group itself, the operation of charge conjugation can be represented by complex conjugation. We may exploit this by explicitly associating charge conjugation $\mathscr{C}$ with the involutary operation of complex conjugation $\left(K_{o}\right)$ for all discussions relevant to the group itself. We are thus led first to the technical problem of determining the charge conjugation matrix $C$ for the various groups under discussion (see Appendix I).

Although the matrix $C$ is completely specified (once definite phase conventions have been stated) there are in general several distinct operators which can equally well be called "the" isoparity $G$. Only after enumerating the complete set of all discrete invariance operators, including physical associations, can a decision as to the "right" isoparity operator be made. In the following we shall only enumerate all of the possible isoparity operators.

We are now in a position to specify the problem more precisely. Given an internal symmetry group $\mathscr{G}$, the charge conjugation operation induces an involutary automorphism on $\mathscr{G}$. Two questions then arise naturally:

a) What are the extended groups, $\mathscr{G}$ ext, each of which includes both the original group $\mathscr{G}$ and the charge conjugation operation?

b) Are there new quantum numbers connected with these extensions?

We may rephrase the first question in this way: Given $\mathscr{G}$ to find all possible groups $\mathscr{G}^{\text {ext }}$ such that $\mathscr{G}^{\text {ext }} / \mathscr{G}=C_{2}$, where the $C_{2}$ group (the cyclic group on two elements) corresponds to the involutary automorphism on $\mathscr{G}$ produced by complex conjugation.

The solution to question a) is developed in Sections II, III and IV and summarized in the Table. Representations of $\mathscr{G}^{\text {ext }}$ are given in Section V. Extensions by involutary operations other than charge conjugation also 
exist and are of importance in discussing the complete set of discrete extensions. We shall only sketch the answer fo this case, giving the results in the Table also.

\section{The adjunction of an involution}

(introductory example)

The problem we seek to solve here has been posed in Section I: Given a symmetry group $\mathscr{G}$ (assumed to be a compact simple Lie group) to determine all groups extended by an involution, $R$, such that $\mathscr{G}^{\text {ext }} / \mathscr{G}=C_{2}$, where $C_{2}$ consists of $R$ and the identity.

Quite generally the elements of the group $\mathscr{G}^{\text {ext }}$ may be written in the form $(a, r)$ where $a$ belongs to $\mathscr{G}$ and $r$ is either the identity $E$ or the involutary automorphism $R$. The product of two elements of $\mathscr{G}^{\text {ext }}$ can be shown to follow the law

$$
\left(a_{1}, r_{1}\right)\left(a_{2}, r_{2}\right)=\left(a_{1} a_{2}^{r_{1}} f_{r_{1}, r_{2}}, r_{1} r_{2}\right)
$$

where $a_{2}^{r_{1}}$ is the element $a_{2}$ of $\mathscr{G}$ transformed by the automorphism associated with $r_{1}$, and the four elements $\left(f_{E}, E, f_{E, R}, f_{R, E}, f_{R, R}\right)$ belong to $\mathscr{G}$. Associativity implies $f_{E, E}=f_{E, R}=f_{R, E}=E$ in $\mathscr{G}$. Two things need then to be determined.

a) the effect of the automorphism $a \rightarrow a^{R}$ in $\mathscr{G}$

b) the element of $\mathscr{G}$ associated with $f_{R, R}$.

In order to simplify the notation let us write

$$
\begin{aligned}
(a, E) & =A \\
(E, R) & =R \\
\left(f_{R}, R, E\right) & =Z=R^{2}
\end{aligned}
$$

then since $E=R R^{-1}=R^{-1} R$ it follows that $f_{R, R}^{R}=f_{R, R}$ and $R^{-1}=\left(f_{R, R}^{-1}, R\right)$ and since

$$
R A R^{-1}=A^{R} \text { and }\left(A^{R}\right)^{R}=A
$$

it follows that $f_{R, R}$ belongs to the center of $\mathscr{G}$. Then also $R^{-1} A R=A^{R}$.

The fact that the internal symmetry group is a Lie group is not essential to the logic of the construction to follow and, for clarity, we may schematize the essentials of the argument by considering an elementary example using the finite cyclic group $C_{4}$, as the "internal symmetry group" $\mathscr{G}$. Adjoining the involution $R$ to $C_{4}$ produces a new group $\mathscr{G}$ ext with 8 elements. There are only 4 finite groups of order 8 with the property that $C_{4}$ is a normal subgroup and $\mathscr{G}^{\text {ext }} / C_{4}=C_{2}$; these yield specific examples of all possible extensions.

a) There are two involutary automorphisms of $C_{4}$ namely

1. $A^{R}=A$ the trivial automorphism

2. $A^{R}=A^{-1}$.

b) What are, in these two cases, the allowed values of $f_{R, R}$ ? 
1. If the automorphism is trivial, define

$$
R^{\prime}=(z, R) \text {, where } z \text { belongs to } C_{4}
$$

$R^{\prime}$ defines the same extension as $R$ but

$$
f_{R, R}^{\prime}=z^{2} f_{R, R} .
$$

This defines two classes of $f_{R, R}$ leading to inequivalent extensions:

Class $\alpha: f_{R, R}$ can be written as a square in the group $C_{4}$.

Class $\beta: f_{R, R}$ cannot be written as a square in the group $C_{4}$.

2. In the second case, where $A^{R}=A^{-1}$, the conditions $f_{R, R}=f_{R, R}^{R}$ $=f_{R, R}^{-1}$ imply that $f_{R, R}$ is a square root of unity in the group $C_{4}$.

Let us next construct the four possible extensions:

Method 1) the direct product:

$$
\begin{aligned}
C_{4}: A^{4} & =E \\
C_{2}: R^{2} & =E, \quad R A R^{-1}=A \\
\mathscr{G}^{\text {ext }} & =C_{4} \otimes C_{2} .
\end{aligned}
$$

Method 2) the direct 'Schreier product' [4], [5]:

$$
\begin{aligned}
C_{4}: A^{4} & =E \\
C_{2}: R^{2} & =A, \quad R A R^{-1}=A \\
\mathscr{G}^{\text {ext }} & =C_{8} .
\end{aligned}
$$

Method 3) the semi-direct product (denoted by (O)) :

$$
\begin{aligned}
C_{4}: A^{4} & =E \\
C_{2}: R^{2} & =E, \quad R A R^{-1}=A^{-1} \\
\mathscr{G}^{\text {ext }} & =C_{4} \odot C_{2}=D_{4} \text { (dihedral group of the square) } .
\end{aligned}
$$

Method 4) the semi-direct Schreier product:

$$
\begin{aligned}
C_{4}: A^{4} & =E \\
C_{2}: R^{2} & =A^{2}, \quad R A R^{-1}=A^{-1} \\
\mathscr{G}^{\operatorname{ext}} & =Q \text { (the quaternion group). }
\end{aligned}
$$

(Note that when $f_{R, R} \neq E, C_{2}$ is not a subgroup of $\mathscr{G}^{\text {ext }}$ but simply a quotient group.)

The essential point for the present paper is the fact that these four techniques (suitably interpreted) for an involutary extension exhaust the possibilities. All four techniques can be combined into two statements:

(a) find all the involutary automorphisms $a \rightarrow a^{r}$ of $\mathscr{G}$. They may be inner or outer i.e. of the form

$$
a^{r}=g_{o} a g_{o}^{-1}
$$

(where $g_{o}$ belongs to $\mathscr{G}$ ) or not of that form, respectively.

(b) find all possible central elements of $\mathscr{G}$ which can be identified with $f_{R, R}$ (remembering that $f_{R, R}^{R}=f_{R, R}$ ). 
The operation $R$ is physically motivated to be a specific symmetry operation (charge reversal) and hence (as discussed in Section $I$ ) must act as a complex conjugation on the group; thus we must take $R=B \mathscr{C}$, where $B$ is any automorphism such that $R$ is involutary. This requirement is not necessarily unique, and there may be several suitable operators $R$, depending on the group $\mathscr{G}$.

One simplification in this general problem exists. We may use the fact that automorphisms $R$ and $R^{\prime}$ which differ by an inner automorphism $\left(R^{\prime}=g_{0} R\right.$ or $R^{\prime}=\left(g_{0}, R\right)$ in a more precise notation) do not define different extended groups. We may thus restrict attention to only those operations $R$ which define distinct groups; this means that various possibilities for $R$ differ by outer automorphisms. In case $\mathscr{C}$ itself induces an inner automorphism (conjugation by $g_{0}$ say) we may use this fact to write the extended group as a direct product (possibly of the Schreier type). Then using the fact that $R$ involves complex conjugation one finds that for the fundamental unitary representation, the central elements are carried into their inverses; this implies that

$$
f_{R, R}=f_{R, R}^{R}=f_{R, R}^{-1}
$$

and that $f_{R, R}$ is the square root of unity in the center of the group $\mathscr{G}$.

The problem of extending $\mathscr{G}$ has thus been reduced to two specific tasks: (1) the determination of all outer automorphisms of $\mathscr{G}$ and (2) the determination of the centre of $\mathscr{G}$; both are classical problems in the mathematical literature. They are discussed in Sections III and IV.

Before leaving the finite group examples, however, it is useful to consider the question: what is "isoparity" for these examples? Since $\mathscr{C}$ is the only non-identical automorphism for $C_{4}$, and is equivalent to $A \rightarrow A^{\prime}=A^{-1}$, it follows that the only extensions containing $\mathscr{C}$ are: $\mathscr{G}^{\mathrm{ext}}=D_{4}(\operatorname{method} 3)$ or $\mathscr{G}^{\mathrm{ext}}=Q(\operatorname{method} 4)$. The isoparity operator $G$ is then just the operation $R$ itself, i.e., $G=\mathscr{C}$. Since $G$ does not commute with all elements of $\mathscr{G}$ ext the isoparity is diagonal only for certain representations of $\mathscr{G}^{\mathrm{ext}}$; in both $D_{4}$ and $Q$ there are four one-dimensional representations for which $G= \pm 1$ and in both one two-dimensional representation for which $G$ is not diagonal ( $G$ parity not defined). This behavior ( $G$ parity $= \pm 1$, or not defined [which we shall call "weak $G$-parity"]) is typical of many of the cases that follow.

\section{Involutary outer automorphisms of $S U_{n}, \bar{R}_{n}, S p_{n}$}

The problem of determining the involutary outer automorphisms of the Lie groups $S U_{n}, \bar{R}_{n}$ (the covering group of the rotation group) and $S p_{n}$ can be put in this way: to determine all transformations $g \rightarrow g^{\prime}$ of the given group $\mathscr{G}$ which leave invariant all defining relations of the 
group, and which are not of the form $g \rightarrow g^{\prime}=g_{o}^{-1} g g_{o}$ (an inner automorphism).

This problem may be transferred to the Lie algebra of the group by noting that every automorphism of the group is an automorphism of the algebra, with infinitesimal inner automorphisms corresponding to derivations of the algebra. Conversely, every automorphism of the algebra corresponds to an automorphism of the covering group. Since the automorphisms of the algebra are given in Ref. [7], our procedure will be to give the explicit transformations for the groups in question, and appeal to the general results for the algebra to prove completeness ${ }^{1}$.

Consider first the unimodular unitary groups $S U_{n}$ which have the vector diagram $A_{n-1}$ for their algebra. The operation of charge conjugation is the automorphism,

$$
g=\exp (i \varphi \cdot X) \rightarrow g^{\prime}=\exp \left(i \varphi \cdot X^{c}\right)
$$

where $X_{A}^{c}=-X_{-A}$. This is an automorphism, since the Lie algebra:

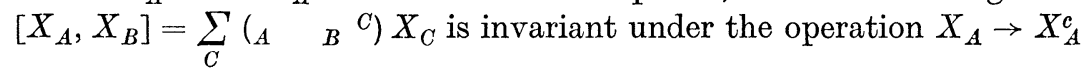
$=-X_{-A}$. Since $S U_{n}$ is simply connected it follows that this automorphism of the algebra is an automorphism of the group. Moreover the operation is clearly involutary; since the classes are not invariant under $\mathscr{C}$ (except for $S U_{2}$ ) it follows that the automorphism is outer for $n \geqq 3$.

From Ref. [7] it is shown that for $S U_{n}, n \geqq 3$, the algebra has only one outer automorphism, which is of period 2 ; this then completes the discussion for $S U_{n}$.

For the groups $\bar{R}_{2 k+1}$ and $S p_{n}$, i.e., the Lie algebras $B_{k}$ and $C_{n / 2}$ respectively, there are no outer automorphisms. This simplifies the extension problem, and shows that $\mathscr{C}$ carries all representations into themselves.

The remaining family of Lie groups, with the covering group $\bar{R}_{2 k}$, all have at least one involutary outer automorphism; the discussion below show that there are three cases to consider:

1 The results given in Ref. [7] can be understood immediately as symmetry operations of the Schouten-Dynkin diagram, which conserve both the type of weight and the connections between them. The Dynkin diagram is composed of the positive simple root vectors, with connections specifying the angle (no connection $\equiv 90^{\circ}$; single line connection $\equiv 120^{\circ}$; double line $\equiv 135^{\circ}$; triple line $\equiv 150^{\circ}$ ) between the joined root vectors (see reference for a full discussion). For the classical groups one has the diagrams:

$$
\begin{aligned}
S U_{n+1} & \Rightarrow A_{n} \\
R_{2 n+1} & \Rightarrow B_{n} \\
S p_{2 n} & \Rightarrow C_{n} \\
& \\
R_{2 n} & \Rightarrow D_{n}
\end{aligned}
$$

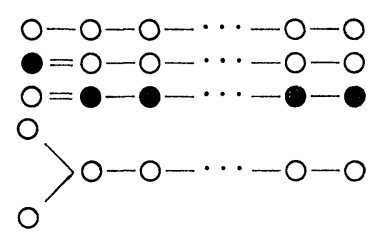


a) the groups $\bar{R}_{4 \ell}$

b) the groups $\bar{R}_{4 \ell+2}$ and

c) the highly exceptional (and therefore very interesting!) case $\bar{R}_{8}$.

Consider first the case $\bar{R}_{4 t}$. The charge conjugation operator, as we shall see in Appendix I, induces the transformation: $\mathscr{C}^{-1} D^{(2 \ell)} \mathscr{C}$ $=C^{-1} D^{(2 \ell)} C$ on the fundamental (spinor) representation $D^{(2 \ell)}$ of dimension $2^{\ell}$, where $C=\prod_{i=1}^{\ell} \Gamma_{2 i-1}$. The charge conjugation matrix $C$ therefore is a product of an even number of $\Gamma_{i}$ matrices; using the fact that $\Gamma_{i} \Gamma_{j}=\exp \left(\frac{\pi}{2} \Gamma_{i} \Gamma_{j}\right)$ and the mapping

$$
\frac{-i}{2} \Gamma_{i} \Gamma_{j} \rightarrow R_{i j}
$$

(the abstract generators of the $\bar{R}_{4 \ell}$ group) one sees that the charge conjugation matrix may be written, in general case, as a product of group elements:

$$
C=\prod_{i=1}^{\ell} \exp \left(i \pi R_{4 i-3,4 i-1}\right) .
$$

It follows that the charge conjugation operation induces an inner automorphism on the group $\bar{R}_{4 \ell}$. The Dynkin diagram shows there exists an outer automorphism for the group: exchange of the two left-hand circles; it is easily seen that this automorphism is generated by the reflection operator, $P=\Gamma_{4 \ell} \Gamma_{4 \ell+1}$.

Let us consider next the rotation covering groups of the form $\bar{R}_{4 \ell+2}$. Just as above we examine the explicit charge conjugation matrix and see that now $C$ consists of a product of an odd number of $\Gamma_{i}$ operators. In this case the charge conjugation matrix must be written as a product of a rotation (i.e., group element) and the reflection operator $P$; that is:

$$
C=P \cdot \prod_{i=1}^{\ell} \exp \left(i \pi R_{4 i-2,4 i}\right)
$$

where $P=\Gamma_{4 \ell+2} \Gamma_{4 \ell+3}$. The charge conjugation operation $\mathscr{C}$ is therefore an outer automorphism for the group $R_{4 \ell+2}$, and from the Dynkin diagram, the only such.

Finally let us examine the exceptional group $\bar{R}_{8}$. It follows from the earlier discussion of $R_{4 \ell}$ that the operation $\mathscr{C}$ is an inner automorphism. From the Dynkin diagram

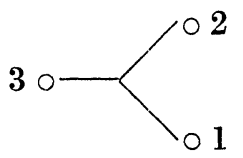

it is clear that the outer automorphisms of the group $\bar{R}_{8}$ (each corresponding to a symmetry of the diagram) form the symmetric group on 3 objects, $S_{3}$. The group $S_{3}$ may be generated by 2 operators $P_{12}$ and 
$P_{23}$, each operator corresponding to the interchange of the numbered circles in the Dynkin diagram. It is quickly seen that one of these operators is the reflection operator, i.e., $P_{12}=\Gamma_{8} \Gamma_{9}$. There are then three involutary outer automorphisms in $\bar{R}_{8}$, generated by the operators $P_{12}, P_{23}$ and $P_{13}=P_{23} P_{12}$.

PaIs [8] has examined this unusual case in some detail and (extending an earlier idea of Tromno [9]; see also [10]) based a dynamical scheme for elementary particles upon its properties. Briefly put the "threefold symmetry" of $\bar{R}_{8}$ allows one to consider the two projected spinors $P_{+} \Psi$ and $P_{-} \Psi\left[\right.$ where $\left.P_{ \pm}=\frac{1}{2}\left(1+P_{12}\right)\right]$, each having eight components, as basis vectors equivalent to the basis vectors of the defining $8 \times 8$ representation. This is a "triality" principle which defines a unique trilinear interaction.

For the present purpose the case $\bar{R}_{8}$ offers and unusual freedom of defining very many (ten!) inequivalent isoparity operators.

\section{The centres of the groups $S U_{n}, \bar{R}_{n}$ and $S p_{n}$}

The remaining task is the determination of the central elements of the various groups. This is easily done if one notes that on every representation the central elements are diagonal, and that on the fundamental representation (the faithful representation of lowest dimension) all central elements are distinct.

For the $S U_{n}$ groups, the fundamental representation is $n \times n$; the central elements are of the form $e^{i \varphi} \mathbf{1}$, and to be unimodular $\varphi$ must be $(2 \pi k / n)$. The centre is therefore $C_{n}$ and is generated by the element: $g_{c}=e^{2 \pi i / n}$. A general irreducible representation is contained in the product of the fundamental representation taken $M$ times, where $M$ is the total number of boxes in the Young diagram characterizing the irreducible representation. The generating element $g_{c}$ of the center is obtained by multiplying $g_{c} M$ times, thus

$$
G_{c}=\exp (2 \pi i M / n) \otimes \mathbf{1} \text {. }
$$

We have thus determined the explicit form of the centre of $S U_{n}$ evaluated on every irreducible representation. We see in particular that this element separates the representation into $n$ types specified by $M$ modulo $n$. This is the generalization of the triality type of $S U_{3}$ [11], [12], [13].

The condition on the possible choices $R^{2}=Z=f_{R, R} \in$ Centre is shown (in section II) to be $R^{-1} Z R=Z$. Under the operation $R=\mathscr{C}$ for $S U_{n}$ the diagonal generators reverse sign; it follows that $R^{-1} g_{c} R$ $=g_{c}^{-1}$ and hence the only admissible elements $Z$ must be of period 2 . This greatly simplifies the extension problem for $S U_{n}$ and shows that $R^{2}=E$ for $S U_{2 k+1}$ and $R^{2}=E$ or $R^{2}=Z\left(Z^{2}=E\right)$ are the only two choices for $S U_{2 k}$. 
Let us turn next to the covering group of the rotation group, $\bar{R}_{n}$. There are two cases: $n$ even and $n$ odd. For the $n=$ odd case the centre has, for the spinor representation, the central element $g_{c}=\exp \left(\pi \Gamma_{1} \Gamma_{2}\right)$ $=-E$ which is of period 2. (This result is seen immediately from the fact that no element other than the identity commutes with all $\Gamma_{i} \Gamma_{j}$.) Since $R^{-1} g_{c} R=g_{c}$, both $g_{c}$ and $E$ are suitable choices for $R^{2}$.

For the group $\bar{R}_{2 k}$ the centre is more complicated. From the fundamental representation one sees that the operators $E$ and $\Gamma_{2 k+1}$ commute with all the generators $\Gamma_{i} \Gamma_{j} i \neq j, i j=1 \ldots 2 k$. Hence the centre contains the four elements $\pm E, \pm \Gamma_{2 k+1}$, with $\Gamma_{2 k+1}^{2}=(-E)^{k}$. One must verify that these elements can be written as group elements in general, i.e., in the form $\exp (i \varphi \cdot X)$. For $[-E]$ one may use simply $\exp \left(\pi \Gamma_{1} \Gamma_{2}\right)$; for $\Gamma_{2 k+1}$ one may use:

$$
\Gamma_{2 k+1}=\prod_{i=1}^{2 k} \Gamma_{i}=\prod_{j=1}^{k} \exp \left(\frac{\pi}{2} \Gamma_{2 j-1} \Gamma_{2 j}\right) .
$$

To simplify the discussion to follow let us denote the group element $\exp \left(\pi \Gamma_{1} \Gamma_{2}\right) \rightarrow \exp \left(2 \pi i R_{12}\right)$ by $A$, and the group element $\Gamma_{2 k+1}$ $=\prod_{j=1}^{k}\left(\exp i \pi R_{2 j-1,2 j}\right)$ by $B$. Then one sees that for $\bar{R}_{4 \ell},\{A\} \otimes\{B\}$ generates the ,Vierergruppe " $C_{2} \otimes C_{2}$, while for $\bar{R}_{4 \ell+2}$, the single element $B$ generates $C_{4}$.

In order to decide which of the elements of the centre are admissible for the square of $R$, that is (confer Section II), the condition: $R^{2}=Z \in$ $\in$ Centre $\rightarrow R^{-1} Z R=Z$, one must next examine the effect of the automorphisms on the centre. There are three cases:

a) $\bar{R}_{4 \ell}:\left(\bar{R}_{8}\right.$ excluded $)$.

The effect of charge conjugation $\mathscr{C}$ is given by the charge conjugation matrix $C$. For $\bar{R}_{4 \ell}$ we recall that $C$ is a group element, hence $\mathscr{C}$ leaves every element of the centre unchanged. Since all elements have period 2, it follows that every element of the centre is admissible.

For the remaining automorphism $P$, one sees that:

$$
\begin{aligned}
P: A & \rightarrow A \\
B & \rightarrow A B \\
A B & \rightarrow B .
\end{aligned}
$$

Thus if we take $R$ to be the automorphism generated by $\mathscr{C} P$, only the element $A$ (and of course $E$ ) is admissible as the square of $\mathscr{C} P$.

b) $\bar{R}_{8}$ :

We have two additional involutary outer automorphisms, $P_{23}$ and $P_{13}$. It is easily seen that the effect of these operations is given by.

$$
\begin{aligned}
P_{12}=P: & B & \rightleftarrows A B \\
P_{23}: & A B & \rightleftarrows A \\
P_{13}: & A & \rightleftarrows B
\end{aligned}
$$

Commun. math. Phys., Vol. 2 
For each operation there is only one admissible element of the centre (besides $E$ ): $A$ for $\mathscr{C} P_{12}=R, B$ for $\mathscr{C} P_{23}$ and $A B$ for $\mathscr{C} P_{13}$.

c) $\bar{R}_{4 \ell+2}$ :

For this case charge conjugation itself generates the only outer automorphism and one finds that under $\mathscr{C}$ the centre $\{B\}=C_{4}$ transforms as $\mathscr{C} \Rightarrow B \rightarrow B^{-1}$. Thus one sees that the only admissible elements of the centre are $E$ and $B^{2}=A$.

For the symplectic group $S p_{n}$ one may use the operators $\mathbf{S}_{(k)}^{q}$ $(k=1,3, \ldots, n-1)$ as the generators of the fundamental $n \times n$ representation, and thereby explicitly obtain the elements of the centre. The only diagonal matrices which are group elements are the identity and the element $g_{c}=\exp \left(2 \pi i S_{(1)}^{0}\right)$ : the centre is the group $C_{2}$. Both elements of $C_{2}$ are easily seen to be admissible for $R^{2}$.

It is helpful to assemble the results of this section in tabular form. This is given in the table. In this table we have also included results for the exceptional groups $G_{2}, F_{4}, E_{6}, E_{7}$ and $E_{8}$.

Table. Involutary automorphisms $R$, centres, admissible elements $Z$ for $f_{R, n}=Z$, types of parities

\begin{tabular}{|c|c|c|c|c|c|}
\hline Groups & Centres & $\begin{array}{c}\text { Charge } \\
\text { conjugation } \\
\text { belongs to }\end{array}$ & $R$ & $Z=f_{R, R}$ & $\begin{array}{l}\text { Number } \\
\text { of inequi- } \\
\text { valent } \\
\text { parities of } \\
\text { each type }\end{array}$ \\
\hline \multirow{2}{*}{$S U_{n}$} & \multirow{2}{*}{$C_{n}=\{A\}$} & $X\left(S U_{2}\right.$ only $)$ & inner & $E, A^{n / 2}$ & 1 or 2 \\
\hline & & $X\left(\right.$ except $\left.S U_{2}\right)$ & outer & $E, A^{n / 2}$ & 1 or 2 \\
\hline $\begin{array}{l}\bar{R}_{2 k+1} \\
(k>0)\end{array}$ & $C_{2}=\{A\}$ & $X$ & inner & $E, A$ & 2 \\
\hline \multirow{2}{*}{$\begin{array}{l}\overline{\bar{R}}_{4 \ell}+2 \\
(\ell>0)\end{array}$} & \multirow{2}{*}{$C_{4}=\{B\}$} & & inner & $E, B, B^{2}$ & 3 \\
\hline & & $X$ & outer & $E, B^{2}$ & 2 \\
\hline \multirow{2}{*}{$\begin{array}{l}\overline{\bar{R}}_{4 \ell} \\
2 \neq \ell>0\end{array}$} & \multirow{2}{*}{$\begin{array}{l}V_{4}=C_{2} \times C_{2} \\
=\{A\} \times\{B\}\end{array}$} & $X$ & inner & $E, A, B, A B$ & 4 \\
\hline & & & outer & $E, A$ & 2 \\
\hline \multirow{4}{*}{$\bar{R}_{8}$} & \multirow{4}{*}{$\begin{array}{l}V_{4}=C_{2} \times C_{2} \\
=\{A\} \times\{B\}\end{array}$} & $X$ & inner & $E, A, B, A B$ & 4 \\
\hline & & & $P_{12}$ (outer) & $E, A$ & 2 \\
\hline & & & $P_{23}$ (outer) & $E, B$ & 2 \\
\hline & & & $P_{13}$ (outer) & $E, A B$ & 2 \\
\hline$S p_{n}$ & $C_{2}=\{A\}$ & $X$ & inner & $E, A$ & 2 \\
\hline$G_{2}$ & $E$ & $X$ & inner & $E$ & 1 \\
\hline$F_{4}$ & $E$ & $X$ & inner & $E$ & 1 \\
\hline \multirow{2}{*}{$E_{6}$} & \multirow{2}{*}{$C_{3}=\{A\}$} & & inner & $E$ & 1 \\
\hline & & $X$ & outer & $\bar{E}$ & 1 \\
\hline$\overline{E_{7}}$ & $C_{2}=\{A\}$ & $X$ & inner & $E, A$ & 2 \\
\hline$\overline{E_{8}}$ & $E$ & $X$ & inner & $E$ & 1 \\
\hline
\end{tabular}




\section{The representations of the extended groups $\mathscr{G}^{\text {ext }}$}

In the preceding three sections we have developed the necessary technical information to carry out explicitly the most general possible adjunction of charge-conjugation $\mathscr{C}$ to an internal symmetry group; the extended group $\mathscr{G}^{\text {ext }}$ is thus completely defined from these results. It is helpful, however, to carry out these results in detail, in order to obtain a clearer view of the isoparity operator $G$. This is most conveniently done by explicitly constructing the representations of $\mathscr{G}^{\text {ext }}$.

Let us assume that the representations of the simple Lie groups ${ }^{2} S U_{n}$, $\bar{R}_{n}, S p_{n}$ are all explicitly available (this is certainly true for $S U_{n}$, and essentially complete for the other cases). Denote these representations by $D^{(a)}(g)$. Under the action of the automorphism $R$ these representations will be carried into another representation which we denote as the conjugate representation $D^{(\tilde{a})}(g)$, to within an equivalence transformation. We have defined as the "charge-conjugation matrix" the matrix $C$ in the equation: $R^{-1} D^{(a)} R=C^{-1} D^{(\tilde{a})} C$, (noting that only for $R=\mathscr{C}$ (the most important case) is this the matrix $C$ of Appendix I.) Finally we denote by $Z$ an admissible distinct element of the centre such that $R^{2}=Z$.

In every case we may then write the representations of the extended group as:

$$
\begin{aligned}
& \mathscr{D}^{[A]}(g, e)=\left(\begin{array}{cc}
D^{(a)}(g) & 0 \\
0 & C^{-1} D^{(\tilde{a})}(g) C
\end{array}\right) \\
& \mathscr{D}^{[A]}(g, r)=\left(\begin{array}{cc}
0 & Z D^{(a)}(g) \\
C^{-1} D^{(\tilde{a})}(g) C & 0
\end{array}\right)
\end{aligned}
$$

If $D^{(a)}$ acts on $\psi, \mathscr{D}^{[A]}$ acts on $\left(\begin{array}{c}\psi \\ \psi^{*}\end{array}\right)$.

There are two possibilities:

(a) If $(a) \neq(\tilde{a})$ (that is, a non-self-conjugate representation of $\mathscr{G}$ ), then $\mathscr{D}^{[A]}$, above, is irreducible. Denote such representations by $\mathscr{D}^{[A, 0]}$.

(b) If $(a)=(\tilde{a})$ (self-conjugate representation of $\mathscr{G}$ ) then $\mathscr{D}[A]$ is reducible and is the sum of two inequivalent representations $\mathscr{D}^{[A+]}$ and $\mathscr{D}^{\left[A^{-}\right]}$. These two representations have the explicit form:

$$
\begin{aligned}
& \mathrm{I}\left\{\begin{array}{l}
\mathscr{D}[A+](g, e)=D^{(a)}(g), \\
\mathscr{D}[A+](g, r)=\varepsilon D^{(a)}(g) C^{-1},
\end{array}\right. \\
& \mathrm{II}\left\{\begin{array}{l}
\mathscr{D}\left[A^{-]}(g, e)=C^{-1} D^{(a)}(g) C,\right. \\
\mathscr{D}\left[A^{-]}(g, r)=-\varepsilon C^{-1} D^{(a)}(g) C \cdot C^{-1},\right.
\end{array}\right.
\end{aligned}
$$

where $\varepsilon \equiv+\left(Z C^{2}\right)^{\frac{1}{2}}, \varepsilon^{4}=E$.

${ }^{2}$ One many discuss (in the physical case, baryonic charge say) the $U_{n}$ group instead of $S U_{n}$, but then the only self-conjugate representations are those with $m_{n n}=0$, i.e., $S U_{n}$. 
These results are obtained using the transformation matrix

$$
\left(\begin{array}{cc}
1 & C \varepsilon^{-1} \\
C^{-1} \varepsilon & -1
\end{array}\right)
$$

provided $C^{4}=E$ (which is the case for charge conjugation).

(Let us note once again that the matrices $Z, C$ are functions of the specific representations involved [i.e., the labels (a)] and moreover $C$ depends upon the automorphism $(R)$.)

The above set of representations is easily seen to define the complete set of irreducible representations of $\mathscr{G}^{\text {ext }}$. There are three types of representations denoted by $[A, 0],[A,+]$ and $[A,-]$.

What now is the extended isoparity operator $G$ ? It is clear from these representations that the isoparity operator $G^{\text {ext }}$ is to be defined now as $G^{\text {ext }}=C R$. This operator is diagonal only for self-conjugate (in $\mathscr{G}$ ) representations. For the remaining representations, the $G^{\text {ext }}$ parity is not defined (that is, $G^{\text {ext }}$ cannot be made simultaneously sharp along with the complete set of observables of $\mathscr{G}$ ). To summarize:

$$
\begin{gathered}
(a)=(\tilde{a}): G^{\text {ext }} \rightarrow \pm 1 \text { for } Z C^{2}=E \\
\rightarrow \pm i \text { for } Z C^{2}=-E \\
(a) \neq(\tilde{a}): G^{\text {ext }} \text { not defined. }
\end{gathered}
$$

The implications of $G^{\text {ext }} \rightarrow \pm i$ have been discussed in reference [14] (see also [15] and the more general discussion of [16]).

\section{The properties of the $G$ parity}

In the preceding sections we have explicitly established the complete set of irreducible representations of the extended group, and have thus, in effect, defined the meaning of $G$ parity in the extended group. The purpose of the present section is to examine the $G$ parity so defined more closely.

There are two possibilities that arise - which we shall call "weak" and "strong" $G$ parity.

(a) Strong G parity.

The simplest possibility occurs for those cases (and only those) where charge conjugation is an inner automorphism $\left(S p_{n}, \bar{R}_{2 k+1}, \bar{R}_{4 \ell}\right)$, which means that all representations are self-conjugate. Taking the operation $R$ to be inner then shows that every representation of the extended group is of the form given in Eq. (V. 3...6), and hence the $G$ parity is well defined:

$$
G|[A \pm]\rangle= \pm \varepsilon|[A \pm]\rangle \text {. }
$$

It is easily shown, using the explicit representations, that this $G$ parity is a conserved multiplicative "parity". This is quite clear in the cases where $\varepsilon^{2} \equiv Z C^{2}=+1$, for in these cases the extended group is a direct product, $\mathscr{G}^{\mathrm{ext}}=C_{2} \otimes \mathscr{G}$, and the $G$ parity is just the group $C_{2}$. 
(This corresponds to the usual $G$ parity for the isospin group, where $G \equiv C^{-1 \mathscr{C}}$ and $G^{2}=+1$. This latter implies that $R^{2}\left(=\mathscr{C}^{2}\right)=C^{2}=Z$, that is, the centre of the isospin group $Z=e^{2 \pi i J}$ is used to make the eigenvalues \pm 1 .) Depending upon whether or not the centre of the symmetry group possesses one or more elements $\varepsilon^{2}$ of period 2 (see the Table) - (remember that $C^{2}$ is always an element of the centre of period 2) one may define other $G$ parities $(G \rightarrow \pm \varepsilon)$ in which $G \rightarrow \pm i$ as well as $G \rightarrow \pm 1$. These parities are also multiplicatively conserved, although this is probably less obvious now since the extended group is no longer a direct product but rather a direct Schreier product (Method 2, Section II). (Recall that $\varepsilon^{2}=Z C^{2}$ is a function of the specific representation $D^{(a)}$ of $\mathscr{G}$ and whether or not $\varepsilon=+i$ or +1 if fully determined from the representations of $\mathscr{G}$ ). The possibility of such a direct Schreier product exists for the $G$ parity in the isospin case; half-integer isospins correspond to $G= \pm i$ and integer isospins to $G= \pm 1$.

(b) Weak G parity

As was seen already in the finite group example of Section II, the $G$ parity may be well defined only on certain representations of the original group $\mathscr{G}$, namely those representations which are self-conjugate. For such representations, the extended representations are of the form of Eqs. (V. 3, . . 6), and correspond to a sharp (strong) G parity $G|[A \pm]\rangle= \pm \varepsilon|[A \pm]\rangle$, where $\varepsilon=\varepsilon([A])$. For the non-self-conjugate representations a strong $G$ parity is not defined.

The crucial question now is this: Is it possible to define a weak $G$ parity which is conserved multiplicatively? One such definition whose suitability we shall examine in a particular case, is this:

(1) For self-conjugate representations such that $\operatorname{tr} C \neq 0$ assign a weak $G$-parity $\varepsilon$ by

$$
\frac{\operatorname{tr} \mathscr{D}[A \pm](e, r)}{|\operatorname{tr} \mathscr{D}[A \pm](e, r)|}= \pm \varepsilon([A]),
$$

which is identical in this case to the strong $G$ parity.

(2) For self conjugate representations such that $\operatorname{tr} C=0$ and for non self conjugate representations, assign as a weak $G$-parity the value zero.

To determine the suitability let us consider all direct products in $S U_{3}$ of the systems $\pm \varepsilon, 0$. It is easily established (using the traces of the representations in Section (V) - note that for $S U_{3}, \operatorname{tr} C$ does not equal zero for self-conjugate representations) that

(a) the direct product, $[A, 0] \otimes[B, 0]$, contains only representations with $G^{\prime}=0$ and/or equal numbers of representations $G^{\prime}=+\varepsilon$ and $G^{\prime}=-\varepsilon$. The direct product, $[A, 0] \otimes[B, \pm]$, also behaves in precisely the same way.

Thus the representations with $G^{\prime}=0$ behave somewhat like a zero in that the $G$ parity of the product adds to zero. 
More difficult to establish, and more interesting, is the rule for a direct product of non-zero $G$ parities. Let us state the rule.

(b) The direct product $\left[A, \varepsilon_{1}\right] \otimes\left[B, \varepsilon_{2}\right]$, where $\varepsilon_{i}= \pm \varepsilon$ contains representations having $\varepsilon_{3}=\varepsilon_{1} \varepsilon_{2}$ and, in addition, the possibility of equal numbers of representations with $\pm \varepsilon_{1} \varepsilon_{2}$, as well as representations with $G^{\prime}=0$.

These rules for the properties of "weak $G$ parity" are not very satisfying from the point of view of defining a quantum number, and show that the most interesting case is probably that of strong $G$ parity. Nevertheless weak $G$ parity does represent a parity-like restriction on the extended group - for one sees that the rules, (a) and (b), state that the weak $G$ parity is conserved in the sense that the product of weak $G$ parities is conserved as a sum of weak $G$ parities. This is a new kind of quantum number which behaves neither additively nor multiplicatively but rather mixes both behaviors. Weak $G$ parity exists formally, as these examples show - whether or nor it is a useful classification remains to be seen. It is useful to note explicitly that the isoparity in $S U_{3}^{\text {ext }}$ is necessarily a weak $G$ parity, with the $F$ and $D$ couplings of octets having $\varepsilon=+$ and $\varepsilon=-$ respectively.

A further discussion of these rules is given in the Appendix II.

Let us note in concluding that in $S U_{4}$ already a very much more complicated behavior occurs, since self-conjugate representations with $\operatorname{tr} C=0$ exist. A weak $G$-parity completely analogous to that of $S U_{3}$ can however, be defined for the factor group $S U_{4} / Z_{4}$.

Acknowledgements. We would like to express our indebtedness to Professor H. Bacry and to Drs. L. Horwitz and N. StraumanN for many illuminating discussions, and to Professor W. T. SHARP for information on several unpublished results on the $S U_{n}$ group. Two of the authors (LCB and JN) would also like to express their gratitude to Professor L. VAN Hove for extending to them the hospitality of the CERN Theoretical Division. A preliminary version of this paper appeared as a CERN report [21]. The hospitality of Professor R. OPPENHEIMER at the Institute for Advanced Study is gratefully acknowledged by J. N.

\section{Appendix I}

\section{The determination of the charge conjugation matrix}

From the discussion in Section I it follows that the effect of the charge conjugation $\mathscr{C}$ on the group $\mathscr{G}$ is, in a particular basis, the same as the effect of complex conjugation. We shall assume the complete set of (unitary) irreducible representations of $\mathscr{G}$ to be known; let us denote one such representation $(a)$ by $D^{(a)}(g)$. Since $(E, \mathscr{C})^{-1}\left(D^{(a)}, E\right)(E, \mathscr{C})$ $=\left(D^{*(a)}, E\right)$, or in a looser notation $\mathscr{C}^{-1} D^{(a)} \mathscr{C}=D^{*(a)}$, and $D^{*(a)}$ is an irreducible representation it must be equivalent to one of the representations in our complete set; we denote this conjugate representation by $(\tilde{a})$. (If $(\tilde{a})=(a)$ the representation is selfconjugate.) Expressing this 
equivalence in symbols, we have:

$$
D^{*(a)}=C^{-1} D^{(a)} C,
$$

which defines (though not uniquely) the general charge conjugation matrix $C$.

There is an alternative, but fully equivalent, view of the matrix $C$ given by the theorem: the matrix $C$ is the Wigner $(1 j)\left(C_{m-m 0}^{j}{ }_{m}^{0}\right.$ for $\left.S U_{2}\right)$ symbol for the representation $D^{(a)}$ of $\mathscr{G}$, that is, the matrix linking states of conjugate representations to form an invariant (scalar) direct product. Note that since the definition, above, of the matrix $C$ is unchanged by a complex phase $e^{i \varphi(a)}$ (which can depend upon the representation), it is clear that conventions are required to make $C$ unique.

The charge conjugation matrix has been discussed for the rotation groups (strictly speaking the covering group of the rotation group) by PaIs [17]; his results are explicit for the fundamental representation. The charge-conjugation matrix for all representations of all the unimodular unitary groups $\left(S U_{n}\right)$ has been given recently by BAIRD and BIEDENHARN [18]. For these groups we may simply take over their results.

For the charge-conjugation matrix of the symplectic group - which is simply connected - one may most economically use an idea due to RACAH and embed $S p(n)$ in the $n \times n$ representation of $S U_{n}$ and thereby obtain the charge conjugation matrix $C$ for the fundamental representation.

Consider the unimodular unitary group $S U_{n}$. The representations are uniquely labelled by a Young frame with $n-1$ rows; that is, by the $n$ integers, $\left(m_{1, n}, m_{2, n}, \ldots, m_{n-1, n}, 0\right)$, with $m_{i, n} \geqq m_{i+1, n}$. The states of an irreducible representation of $S U_{n}$ may themselves be labelled uniquely by a Young tableau (filling in the Young frame lexically but allowing repetitions), or equivalently by a Gelfand pattern:

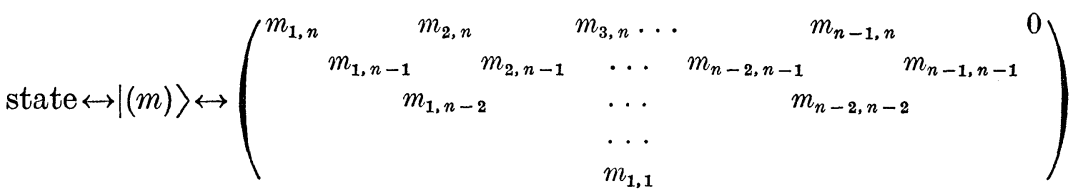

where $(m)$ denotes $\left(m_{i, j}\right)$ and $m_{i, j+1} \leqq m_{i, j} \leqq m_{i+1, j+1}$.

The conjugation operation acting on a representation $\left(m_{i, n}\right)$ carries it into a representation equivalent to the representation $\left(\tilde{m}_{i, n}\right)$, with $\tilde{m}_{i, n}=m_{1, n}-m_{n-i+1, n}$. The state $\left(m_{i, j}\right)$ is carried into the state $\tilde{m}_{i, j}=m_{1, n}-m_{j-i+1, j}$. (Note that $(\tilde{m})$ is unimodular.) It is shown in Ref. [18] that the matrix $C$ is given by

$$
C_{(\tilde{m}),(m)} \equiv(-)^{\frac{(n+1)(n+2)}{2}}(-)^{\varrho[(m)]}(-)^{\frac{n(n+1)}{2}} m_{1, n} \delta_{(m)}^{(\tilde{m})}
$$


where $\varrho[(m)] \equiv \sum_{j=1}^{n-1} \sum_{i=1}^{j} m_{i, j}$. (Note that $C$ is arbitrary to an over-all sign which may be interpreted as an intrinsic $G$ parity. With the convention chosen here, the states belonging to the centre of the weight diagram of the adjoint representation $\left(S U_{n}\right)$ have always $C=1$.)

The formal properties of matrix $C$ are now seen to be:

$$
\begin{aligned}
& C^{*}=C \\
& C^{t}=(-) \frac{n(n-1)}{2} m_{1, n} C \\
& C^{\dagger}=C^{-1} .
\end{aligned}
$$

(Note that in general $C$ connects states of different representations.)

For the adjoint representation $(211 \ldots 10)$, which is self-conjugate, the matrix $C$ can be considered to act on the generators themselves. That is, introducing the generators $X_{A}$,

$$
C^{-1} \exp (i a \cdot X) C=\exp \left(-i a \cdot X^{t}\right) \text {. }
$$

Since this is true for every $a_{A}$, it follows that:

$$
C^{-1} X_{A} C=-X_{A}^{t}=-X_{-A} \text {. }
$$

This relation, which is independent of the phase conventions on $C$ for self-conjugate representations, can be taken as the defining property instead of defining $C$ to be the Wigner " $(1 j)$ " symbol.

The matrix $C$, considered as a $(1 j)$ symbol, has the property mentioned earlier that it defines an invariant product of wave functions. Let $\psi_{1} \in(m)$ and $\psi_{2} \in(\tilde{m}) \equiv\left(m^{\prime}\right)$. Then the product:

$$
I=\psi_{1} C \psi_{2}=\sum_{(m),\left(m^{\prime}\right)} \psi_{1}^{(m)} C_{(m),\left(m^{\prime}\right)} \psi_{2}{ }^{\left(m^{\prime}\right)}
$$

is an invariant under the action of the group generators, $X_{A}$ (assuming commutation of the $\psi$ 's). The matrix $C$ is itself an invariant, in the sense that:

$$
C^{\prime} \equiv S^{t} C S=C
$$

for all $S, S=\exp (i a \cdot X)$; that is, for all unitary transformations of the group $S U_{n}$. (These results follow immediately from Ref. [6]).

It is interesting to note that if $\psi_{1}=\psi_{2}$ (and hence we have a selfconjugate representation), then the invariant vanishes identically for $C^{t}=-C$. For this condition to hold, we must have $(-)^{\frac{1}{2} n(n-1) m_{1, n}}=(-)$, which in turn requires: (a) $m_{1, n}=$ odd integer and (b) $n=2,3 \bmod 4$, as well as (c) $n=0 \bmod 2$ (from the requirement that $(\tilde{m})=(m)$ ). Hence we see that there exists no non-vanishing invariant, $\left(\psi^{t} C \psi\right)$, for the representations $(\tilde{m})=(m), m_{1, n}=$ odd in $S U_{4 k+2}$. This is a familiar property of the half-integer representations in $S U_{2}$.

Let us next extend this result to the defining representation of rotation and symplectic groups. In order to use the results obtained above 
let us use the technique of embedding $R_{2 k+1}$ in $S U_{2 k+1}$ and $S p_{2 k}$ in $S U_{2 k}$. The generators of $S U_{n}(n=2 k$ or $2 k+1)$ are maps of the specific generators defined in the $n \times n$ representation. Rather than the Weyl form $X_{A}$ let us now use Racah's form [19] based on the tensor operators $\mathbf{S}_{(k)}^{q}(k=1,2, \ldots, n-1)$ defined on the $n$-dimensional angular momentum space having $j=\frac{n-1}{2}$. [The operators $\mathbf{S}_{(k)}^{q}$ are (to a constant factor) the matrix operators given by the Wigner coefficients $C_{m}^{j} \underset{q}{k} \underset{m}{j}$, acting in the $n \times n$ dimensional space of the fundamental representation.]

Now the charge conjugation matrix $C$ is defined for a definite group, and exists as a specific matrix for each representation. But different groups may have representations (reducible or irreducible) of the same dimensionality, and for this case the possibility arises of investigating the action of different charge conjugation matrices on the same abstract space. This possibility thus affords an interesting method of splitting a group into a subgroup, as the following example shows.

Under the action of the $S U_{n}$ matrix $C$ we have the relation:

$$
C_{(n)}^{-1} \chi_{A} C_{(n)}=-\chi_{-A}
$$

just as before, (since $\chi_{A}$ are simply specific matrices, the $X_{A}$ for $n \times n$ representation). Now let us ask: what is the effect of the $S U_{2}$ chargeconjugation matrix $C_{(2)}$ in the representation of dimension $n$ on the generators $\chi_{A}$ ? Since $\chi_{A}=\mathbf{S}_{(k)}^{q}$, it is clear that we seek: $C_{(2)}^{-1} \mathbf{S}_{(k)}^{q} C_{(2)}$. Now the charge-conjugation matrix in $S U_{2}$ is simply $\left[C_{(2)}\right]_{m^{\prime}, m}$ $=(-)^{j-m} \delta_{m}^{-m}$, as follows from the general results earlier ${ }^{3}$. One cannot conclude that, for the mapping $\mathbf{S}_{(k)}^{q} \leftrightarrow|k, q\rangle$, the phase is $(-)^{k-q}$, since the consistency of the $X_{A}$ phase relations and the $\mathbf{S}_{(k)}^{q}$ phase relations must be established. The required phase relation is that:

and hence:

$$
\left|\mathbf{S}_{(k)}^{q}\right\rangle=\left\{\begin{array}{l}
(-)^{q}|k, q\rangle \quad q>0 \\
|k, q\rangle \quad q \leqq 0
\end{array}\right.
$$

$$
C_{(2)}^{-1} \mathbf{S}_{(k)}^{q} C_{(2)}=(-)^{k} \mathbf{S}_{(k)}^{-q} .
$$

In order that this transformation be an automorphism for a Lie algebra generated by the $\mathbf{S}_{(k)}^{q}$, it is then required that $(-)^{k}=-1$. We have thus extracted from the $S U_{n}$ group the subgroup ${ }^{4}$ generated by the operators $\left\{\mathbf{S}_{(k)}^{q}\right\}, k=1,3, \ldots\left\{\begin{array}{l}n-1 n=\text { even } \\ n-2 n=\text { odd. }\end{array}\right.$ For $n=2 k$, this is the symplectic group $S p_{k}$; for $n=2 k+1$ this is the rotation group $R_{2 k+1}$.

3 Actually $(-)^{j+m}$ results, but the difference is unimportant for what follows.

4 This stems from RACAH [19], who obtained it by direct computation from the commutation relations. The method used above shows that the underlying principle is more general. 
It is now unnecessary to calculate the explicit matrices $C$ for the defining representation of the $R_{2 k+1}$ and $S p_{k}$ groups - since this matrix is simply the matrix $C_{(2)}$ of appropriate dimension, $n=2 j+1$. It is clear also that the matrices $C$ have precisely the desired general properties:

and

$$
C^{*}=C, \quad C^{\dagger}=C^{-1}
$$

$$
C^{-1} \chi_{A} C=-\chi_{-A}=-\chi_{A}^{t},
$$

(where now $\chi_{A}$ refers to the Cartan form of the $R_{2 k+1}$ or $S p_{k}$ generators).

For the particular case of the fundamental $(n \times n)$ representation, we have the result:

using $n=2 j+1$.

$$
C^{t}=(-)^{n-1} C
$$

Hence for the rotation group $R_{2 k+1}$ we get: $C^{t}=C$ and for the symplectic group, $C^{t}=-C$. The significance of the matrix $C$ as defining an invariant now shows that the defining representation involves a quadratic symmetric real form for the rotation group, and antisymmetric, real form for the symplectic group. The $R_{2 k}$ result also follows, by embedding it in $R_{2 k+1}$.

Rather more interest attaches to the spinor representations of $R_{n}$. The charge conjugation matrices for these can be obtained by appropriately specializing the general results. It is precisely these cases, however, that have been treated by PAIS [17], and we can avail ourselves of his results. Pars finds that: for $n=2 \ell$ and $n=2 \ell+1$, the $2^{\ell}$ component spinor representation $\psi$ of $\bar{R}_{n}$ is transformed according to

$$
\begin{gathered}
\psi^{\prime}=S \psi \quad S=1+\frac{1}{4} \varepsilon_{\mu \nu} \Gamma^{\mu} \Gamma^{\nu} \\
\Gamma_{\mu} \Gamma_{\nu}+\Gamma_{\nu} \Gamma_{\mu}=2 S_{\gamma \nu} 1 \\
\Gamma_{\mu}^{\prime}=S^{-1} \Gamma_{\mu} S \quad(\mu, \nu=1 \ldots n) .
\end{gathered}
$$

The matrix $C$ is again defined in such a way that

and $\left(\psi^{t} C \psi\right)$ is invariant.

$$
C^{\prime}=S^{t} C S=C
$$

Choose a representation of $\Gamma_{\mu}$ such that $\Gamma_{\mu}^{\dagger}=\Gamma_{\mu}$ and $\Gamma_{2 \mu-1}^{t}=\Gamma_{2 \mu-1}$, $\Gamma_{2 \mu}^{t}=-\Gamma_{2 \mu}$. Then one gets for $C$ :

$$
\begin{array}{lll}
n=2 \ell & C=\prod_{\alpha=1}^{\ell} \Gamma_{2 \alpha-1}, \quad C^{t}=(-)^{\frac{\ell(\ell-1)}{2}} C \\
& C \Gamma_{\alpha} C^{-1}=(-)^{\ell+1} \Gamma_{2}^{t}, \quad \alpha=1, \ldots 2 \ell \\
n=2 \ell+1 & C=\prod_{\alpha=1}^{\ell+1} \Gamma_{2 \alpha-1}, \quad C^{t}=(-)^{\frac{\ell(\ell+1)}{2}} C & \\
& C \Gamma_{\alpha} C^{-1}=(-)^{\ell} \Gamma_{\alpha}^{t}, & \alpha=1 \ldots 2 \ell \\
& \Gamma_{2 \ell+1}=(-i)^{\ell} \prod_{\alpha=1}^{2 \ell} \Gamma_{\alpha} .
\end{array}
$$


Let us note for clarity, that - as PaIs has discussed - the spinor representation for $n=2 \ell$ is not irreducible if the group does not contain reflections, (the reflection operator can be taken as $\Gamma_{1} \Gamma_{2 \ell+1}$ ). In the absence of reflections, the spinor representation of $\operatorname{dim} 2^{\ell}$ splits into two representations of dimension $2^{\ell-1}$ (conjugate under reflections).

\section{Appendix II}

We shall prove here the validity of the "weak $G$ parity" conservation rules only for the $S U_{3}$ case, since this is typical of the considerations that enter.

One needs only to consider explicitly the direct product of the group

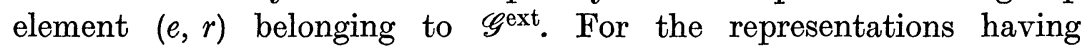
$G^{\prime}= \pm \varepsilon \equiv \varepsilon_{1}$ (which requires that $[A]=[\widetilde{A}]$ the element $(e, r)$ corresponds to a matrix whose trace is

$$
\operatorname{tr}\left(\mathscr{D}^{\left[A, \varepsilon_{1}\right]}(e, r)\right)=\varepsilon_{1} \operatorname{tr}\left(C^{-1}\right) .
$$

(The matrix $C$ has been explicitly given in Appendix I.)

Using the fact that every self-conjugate $S U_{3}$ representation has the form: $[A]=[2 k k 0]$, we find that

$$
\operatorname{tr}\left(C^{-1}\right)=(-)^{k}(k+1)
$$

(This result can be interpreted - aside from sign - as the number of self-conjugate states belonging to the representation.)

Consider next the direct product $([A] \equiv[2 k k 0]$ and $[B] \equiv[2 \ell \ell 0])$ :

$$
\begin{aligned}
\operatorname{tr}\left[\mathscr{D}^{\left[A, \varepsilon_{1}\right]}(e, r)\right. & \left.\otimes \mathscr{D}^{\left[B, \varepsilon_{2}\right]}(e, r)\right] \\
& =\left(\operatorname{tr} \mathscr{D}^{\left[A, \varepsilon_{1}\right]}(e, r)\right) \cdot\left(\operatorname{tr} \mathscr{D}^{\left[B, \varepsilon_{2}\right]}(e, r)\right) \\
& =\varepsilon_{1}(-)^{k}(k+1) \cdot \varepsilon_{2}(-)^{\ell}(\ell+1) .
\end{aligned}
$$

To proceed further we must recall that for $S U_{3}$ the direct product $[2 k k 0] \otimes[2 \ell \ell 0]$ contains the self-conjugate representation [ $2 m \mathrm{~m} 0$ ] with $m=k+\ell, k+\ell-1, \ldots, k-\ell$ with the corresponding multiplicities $1,2,3, \ldots, \ell+1, \ell, \ldots, 2,1$.

Since the non-self-conjugate representations contained in the direct product have zero trace (for the element $(e, r)$ ), we must satisfy the equation (taking $k \geqq \ell$ ):

$$
\varepsilon_{1} \varepsilon_{2}(-)^{k+\ell}(k+1)(\ell+1)=\sum_{\substack{m=k-\ell \\ \text { with } \\ \text { repetitions }}}^{k+\ell} \varepsilon_{m}(-)^{m}(m+1),
$$

where the repetitions correspond to the multiplicity given above. By induction, one shows that the solution is given by $\varepsilon_{m}(-)^{m}=\varepsilon_{1} \varepsilon_{2}(-)^{k+\ell}$ for the first occurrence of the representation [ $2 m m 0$ ] with the remaining occurrences taking the signs $-\varepsilon_{m},+\varepsilon_{m}, \ldots$. 
Thus the terms in the sum cancel in pairs, and only the representations with odd multiplicity survive, each once. The result is the identity, familiar from the addition of angular momenta, that:

$$
\begin{aligned}
\varepsilon_{1} \varepsilon_{2}(-)^{k+\ell} & (k+1)(\ell+1) \\
& =\varepsilon_{k+\ell}(-)^{k+\ell}(k+\ell+1)+ \\
& +\varepsilon_{k+\ell-2}(-)^{k+\ell}(k+\ell-1)+\cdots+\varepsilon_{k-\ell}(-)^{k+\ell}(k-\ell+1) \\
& =(-)^{k+\ell} \varepsilon_{f} \sum_{i=0}^{2 \ell}(k-\ell+1+2 i)=(-)^{k+\ell} \varepsilon_{f}(k+1)(\ell+1) .
\end{aligned}
$$

Thus $\varepsilon_{f}=\varepsilon_{1} \varepsilon_{2}$, i.e., the first occurrence of the representations [ $2 m m 0$ ] have the same $G$ parity, which is conserved multiplicatively for these representations. The remaining representations have either $G$ parity $=0$ or contain equal numbers of $G$ parities $\pm \varepsilon_{1} \varepsilon_{2}$.

This establishes rule (b) of Section (VI) for the $S U_{3}$ case, and thus completes the proof of the weak $G$ parity rules for the extension $\mathscr{G}^{\text {ext }}$ of $S U_{3}$. (The $G$-parity for $S U_{3}$ has also been discussed in reference [20].)

\section{References}

[1] Michel, L.: Nuovo Cimento 10, 319 (1953).

[2] LeE, T. D., and C. N. YANG: Nuovo Cimento 13, 749 (1956).

[3] Miches, L.: Lectures "Invariance in Quantum Mechanics and Group Extensions", Istanbul (1962); preprint version Ecole Polytechnique, Paris. Published in Group Theoretical Concepts and Methods in Elementary Particle Physics. Ed. F. GÜrsey, Gordon and Breach. New York 1964.

[4] Schreier, O.: Monatsh. Math. Physik 34, 165 (1926).

[5] - Abhandl. math. Seminar hamburg. Univ. 4, 321 (1926).

[6] HaLL, M.: The theory of groups. New York: Macmillan 1959.

[7] SalaM, A.: Lecture in "Trieste Seminar in Theoretical Physics", p. 173, Int. Atomic Energy Agency, Vienna (1963).

[8] PaIs, A.: Phys. Rev. Letters 7, 291 (1961).

[9] Tromno, J.: Nuovo Cimento 6, 69 (1957).

[10] - Lecture in "Trieste Seminar in theoretical physics", p. 251, Int. Atomic Energy Agency, Vienna (1963).

[11] Fowler, E. C., and L. C. Biedenharn: Nuovo Cimento 33, 1329 (1964).

[12] Simoni, A., and B. Vitale: Nuovo Cimento 33, 1199 (1964).

[13] BaCRY, H., J. Nuyts, and L. van Hove: Phys. Letters 12, 285 (1964).

[14] Yang, C. N., and J. Tiomno: Phys. Rev. 79, 495 (1950).

[15] Wick, G. C., A. S. Wightman, and E. P. Wigner: Phys. Rev. 88, 101 (1952).

[16] JАUCH, J. M., and B. Misra: Helv. Phys. Acta 34, 699 (1961).

[17] PaIs, A.: J. Math. Phys. 3, 1135 (1962).

[18] BaIRd, G. E., and L. C. Biedenharn: J. Math. Phys. 5, 1723 (1964).

[19] RACAH, G.: Princeton Lectures (1951).

[20] Dothan, Y.: Nuovo Cimento 30, 399 (1963).

[21] Biedenharn, L. C., J. NuYTs, and H. RuEGG: "On the generalization of the isoparity", CERN 65-3 (11 Jan. 1965). 